\title{
Electromagnetic Scattering Models for Ocean Remote Sensing: Overview and Guide to Use
}

\author{
Gerardo Di Martino, Antonio Iodice, Daniele Riccio, Giuseppe Ruello \\ Dept.of Electrical and Information Technology Engineering \\ University of Napoli Federico II, \\ Napoli, Italy
}

\begin{abstract}
Several microwave sensors are currently employed to monitor the oceans. In order to obtain the desired information from these sensors' data, models describing how sea state parameters are related to the backscattered signal are needed. This is achieved by electromagnetic scattering models based on reliable sea surface models. In this work, we review the main scattering models and suggest the proper one for each of the main sensors and marine applications.
\end{abstract}

Keywords—Remote Sensing; electromagnetic models.

\section{INTRODUCTION}

The ocean surface is continuously monitored by microwave sensors. Altimeters are used to measure the average sea level, and to monitor significant wave height and wind intensity; scatterometers are mainly employed to monitor wind intensity and direction; and synthetic aperture radars (SAR), with their high-resolution capability, can be used for sea spectrum measurement, for ship detection, and for oil spill detection; finally, many of the above cited applications can be also implemented by using coastal or marine radars, although with a more limited coverage.

The extraction of meaningful information from the measured electromagnetic field requires the comprehension of the interaction between electromagnetic fields and ocean surfaces. Therefore, in Section II we present a review of the most used sea surface statistical models; in Section III we present the most used electromagnetic scattering methods; and in Section III we provide a link between the introduced models and their use in the application of actual sensors.

\section{Sea Surface Models}

Evaluation of scattering from the ocean requires a sea surface statistical model and a method for the computation of scattering. With regard to the former, the surface spectrum (i.e., its power spectral density) is employed: for a fully developed sea, the Pierson-Moskowitz spectrum [1] can be used, whereas the JONSWAP spectrum [2] accounts for the case of a limited fetch.
In particular, the Pierson-Moskowitz spectrum $S^{P M}$ can be expressed as a function of the sea wavenumber $k$ :

$$
S^{P M}(k)=\frac{2 \pi a}{k^{3}} e^{\frac{-0.74 g^{2}}{k^{2} u_{195}^{4}}}
$$

where $g$ is the gravitational acceleration $\left(9.8 \mathrm{~m} / \mathrm{s}^{2}\right), u_{19.5}$ is the wind speed measured at $19.5 \mathrm{~m}$ height $\left(u_{19.5} \cong 1.026 u_{10}\right)$ and $a=4.05 \cdot 10^{-3}$.

The JONSWAP empirical spectrum $S^{J}$ can be expressed as:

$$
S_{k}^{J}(k)=\frac{3.3^{r} \pi \alpha}{k^{3}} e^{-\frac{5}{4}\left(\frac{k_{p}}{k}\right)^{2}}
$$

where $r, \alpha$ and $k_{p}$ can be evaluated as a function of the gravitational velocity, the wind speed measured at $10 \mathrm{~m}$ height, the Fetch and some empirically evaluated parameters.

However, both of these models do not provide an accurate description at high spatial frequencies, i.e., for the gravitycapillary and capillary wave ranges, which greatly influence microwave scattering especially at intermediate incidence angle. Accordingly, extensions of these models have been developed: the modified Pierson-Moskowitz spectrum [1] and the Elfouhaily spectrum $S^{E}$ [2]:

$$
S_{k}^{E}(k)=\frac{c\left(k_{p}\right)}{c(k)} S_{k}^{J}(k)+\frac{c_{m}}{c(k)} \frac{\pi \alpha_{m}}{k^{3}} e^{-\frac{1}{4}\left(\frac{k}{k_{m}}-1\right)^{2}} .
$$

The Elfouhaily spectrum is expressed as a variant of the JONSWAP spectrum in terms of empirical parameters $\left(c_{\mathrm{m}}, \alpha_{\mathrm{m}}\right.$ and $k_{\mathrm{m}}$ ). Both models lead to a range of spatial frequencies in which a power-law spectrum is obtained that can be fruitfully interpreted in terms of fractal geometry, as shown in Fig. 1 [38].

Finally, methods to evaluate the modifications on the sea spectrum caused by a surface oil film ("capillary wave damping") have been devised [1]. 


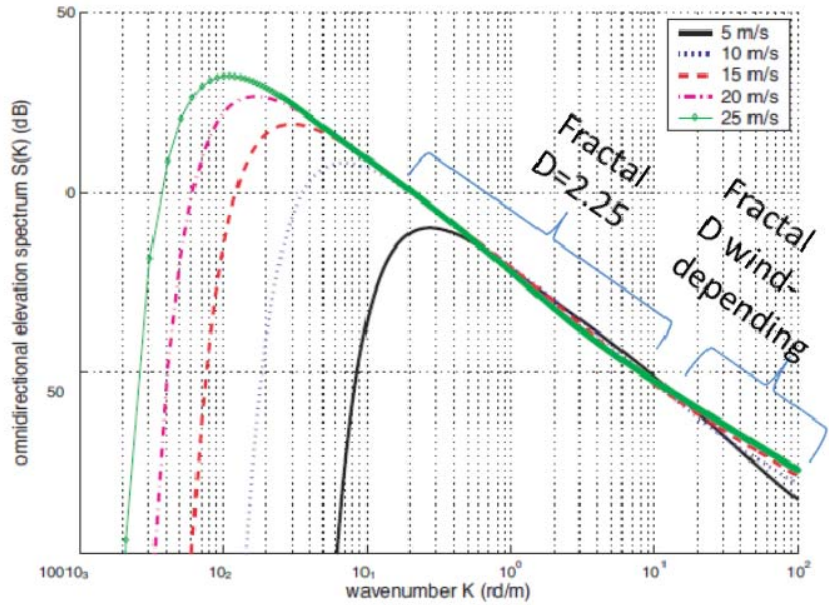

Fig. 1. Elfouhaily spectrum for different wind speeds and fetch $F=100$ $\mathrm{km}$.

\section{ELECTROMAGNETIC METHODS}

With regard to the methods for the computation of scattering, the most widespread are the Kirchhoff approach (KA) in its geometrical-optics (GO) or physical-optics (PO) approximations [3-6], and the small perturbation method (SPM) [3-4,7]. The former holds for high frequencies or large roughness (i.e., roughness standard deviation of the order of, or much larger than, electromagnetic wavelength), and for small to intermediate incidence angle. The SPM holds for low frequencies or small roughness, and for intermediate and large incidence angle. The advantage of these methods is the simplicity of their formulation, but their drawback is their limited ranges of validity. In particular, both methods do not account for multiple scattering and shadowing, so that they cannot be employed for near-grazing angle incidence, and they do not model cross-polarisation and de-polarisation effects. The main properties of these two methods are summarized in Table I.

TABLE I. SUMMARY OF KA AND SPM PROPERTIES

\begin{tabular}{|l|c|c|}
\hline \multicolumn{3}{|c|}{ Main Properties } \\
\hline Property & $\boldsymbol{K} \boldsymbol{A}$ & $\boldsymbol{S P \boldsymbol { M }}$ \\
\hline Vormulation & simple & Simple \\
\hline Multiple scattering & $\begin{array}{c}\text { high roughness } \\
\text { and small to } \\
\text { moderate } \\
\text { incidence angles }\end{array}$ & $\begin{array}{c}\text { small roughness } \\
\text { and intermediate } \\
\text { incidence angles }\end{array}$ \\
\hline Shadowing & NO & No accounted for \\
\hline Cross-polarization & NO & NO \\
\hline De-polarization & NO & NO \\
\hline
\end{tabular}

In order to obtain a method whose range of validity is the union of those of KA and SPM, two-scale models (TSM) have been developed [9]. In this approach, we consider the ocean surface as composed of a large-scale component with a superimposed small-scale roughness, as shown in Fig. 2. The large-scale roughness is locally treated by replacing the surface with a slightly rough tilted facet, whose slope is the same of the smoothed surface at the center of the pertinent facet. Therefore, the overall scattering is decomposed as the sum of scattering from large-scale roughness, computed via KA, and from smallscale roughness, computed via SPM.

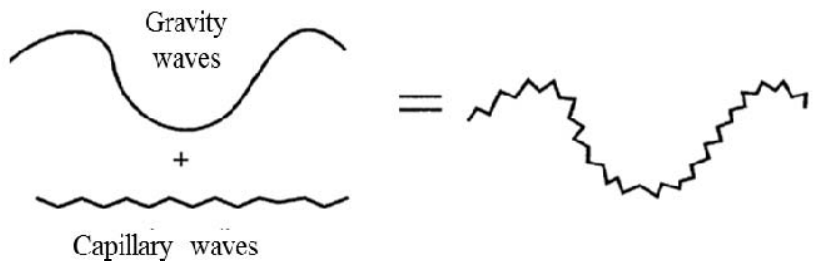

Fig. 2. TSM ocean surface description

A polarimetric version of the TSM, the PTSM [9], also allows to account for cross-polarisation and de-polarisation effects. In the PTSM, the scattering surface is described as a collection of randomly tilted facets, representing the large-scale roughness, over which a small-scale roughness is superimposed. The random tilt is described by a random rotation of the local incidence plane and, as a consequence, of the local incidence angle $l$.

Accordingly, scattering at intermediate incidence angle is computed as the SPM scattering from a collection of tilted facets:

$$
\sigma_{p q}^{0}=\frac{4}{\pi} k^{4} \cos ^{4} \vartheta_{l}\left|\chi_{p q}\right|^{2} S\left(2 k \sin \vartheta_{l}, 0\right)
$$

where

$$
\underline{\underline{\chi \chi}}\left(\vartheta_{l}, \beta\right)=\underline{\underline{R_{2}}}(\beta) \cdot\left(\begin{array}{cc}
\alpha_{h}\left(\vartheta_{l}\right) & 0 \\
0 & \alpha_{v}\left(\vartheta_{l}\right)
\end{array}\right) \cdot{\underline{\underline{R_{2}}}}^{-1}(\beta)
$$

and

$$
\underline{\underline{R_{2}}}(\beta)=\left(\begin{array}{cc}
\cos \beta & \sin \beta \\
-\sin \beta & \cos \beta
\end{array}\right)
$$

where $\beta$ is the rotation of the local incidence plane.

If both large and small scale roughness are within a sensor resolution cell (altimeters and scatterometers), then overall scattering can be computed by averaging SPM scattering over facets' slopes. Conversely, if small scale roughness is within a resolution cell, but large scale roughness is larger than resolution cell (this is what typically happens for SAR images of the ocean surface), then NRCS from different resolution cells changes according to the local slope, i.e., to the tilt of the cell mean plane. 
An example of the NRCS evaluated with the PTSM method is proposed in Fig. 3, where the electromagnetic wavelength is about $5 \mathrm{~cm}$ (L-band), and the sea surface roughness is evaluated with fractal models.

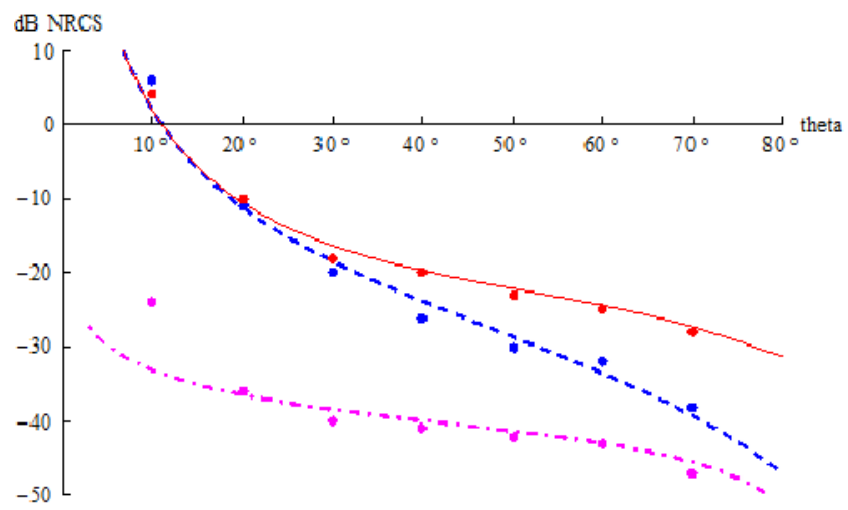

Fig. 3. Measured (dots) and PTSM (lines) backscattering coefficients at VV (solid line), HH (dashed line) and HV (dot-dashed line) polarizations.

Note that the "tilt modulation" is the effect which renders the wave pattern clearly visible on SAR images, as presented in Fig.4.

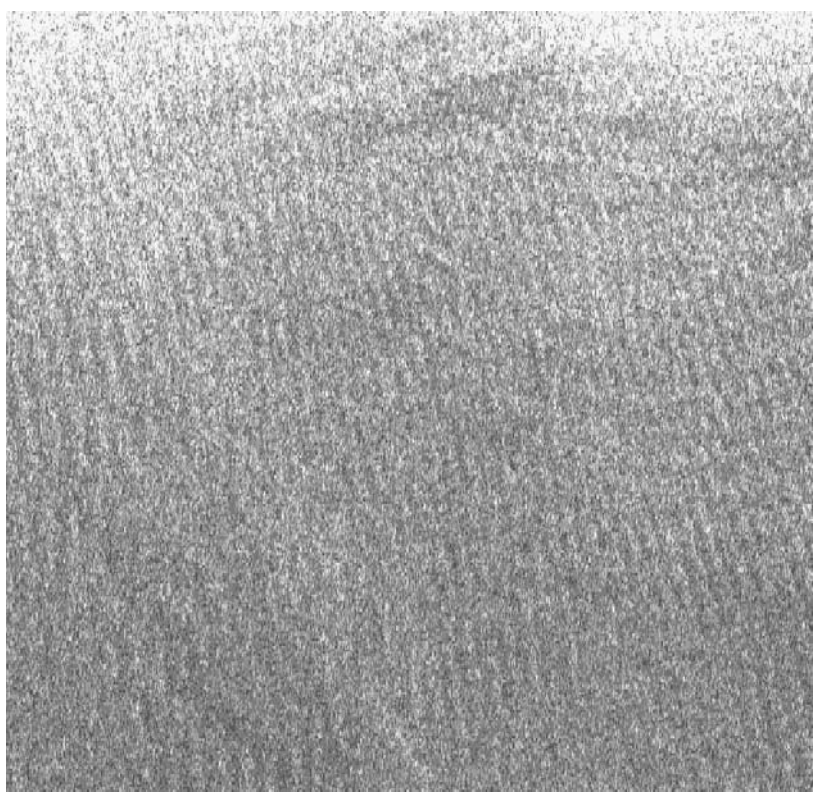

Fig.4: SAR image of a sea surface

Finally, a very wide range of validity, also including neargrazing angle incidence, is obtained by numerical methods based on the method of moments (MoM) and Monte Carlo simulations [8]. The typical procedure required at least the following steps:

a. Generation of e a large number of surface realizations. b. For each surface realization, computation of surface fields via method of moment.

c. For each surface realization, computation of the scattered field via numerical integration.

d. Average of the obtained scattered fields and squared modula of scattered fields

However, the advantages of numerical methods are balanced by the very high computational time and the fact that there is a lack of physical insight.

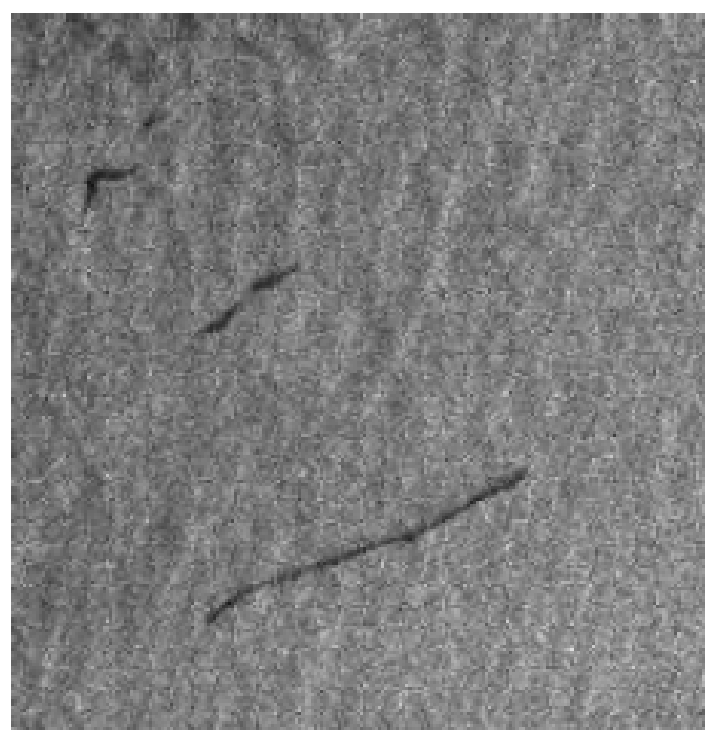

Fig. 5. SAR image of a sea surface covered by oil spills

\section{OCEAN REMOTE SENSNIG}

The considerations introduced in the previous sections allow to identify the different models that can be suggested for different sensors. For the altimeter, in view of its small incidence angle, the KA can be used, and the classical PiersonMoskowitz (ocean) or JONSWAP (sea) spectra may be sufficient. For scatterometer and SAR, with their intermediate incidence angle, Modified Pierson-Moskowitz (ocean) or Elfouhaily (sea) spectra should be used, and TSM or PTSM (i.e., SPM from randomly tilted facets) are suitable for scattering evaluation.

The presence of oil spills on the ocean surface can be evaluated by considering the modifications of the ocean spectrum due to surfactant materials [1]. The corresponding electromagnetic models found meaningful use in the comprehension of the potentiality of SAR sensors for extracting information on the presence of oil on the ocean surface. In addition, the electromagnetic models allow to analyze the similarities and differences between different kind of slicks. Typical problems are related within the discrimination of oil slicks from look-alikes. In Fig. 5 the 
effect of oil spills on the ocean surface are displayed. The corresponding SAR image is characterized by areas of low backscatter, due to damping of capillary waves under the action of the oil. An important characteristics of the slicks displayed in Fig. 5 is their geometrical regularity, which is an index of the fact that they have been provoked by human action. This consideration suggests to measure the regularity of the slick border in order to discriminate between natural oil or natural look alikes (as a local lack of wind) and spills due to human action.

The electromagnetic models have been used in conjunction with a SAR raw signal simulator for producing simulation of oil slicks, lack of wind and natural slicks with fractal borders. An example of simulation of an oil slick and a lack of wind is proposed in Fig. 6.

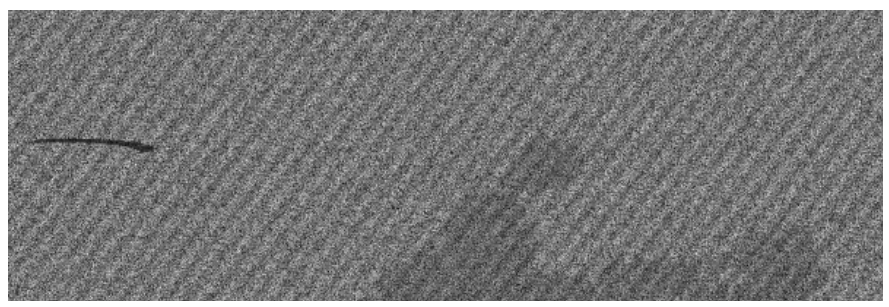

Fig. 6. Simulated SAR image relative to human action (on the left), and a lack of wind (on the right).

Finally, for coastal and marine radar, in view of their neargrazing angle incidence, numerical methods are most appropriate.

\section{CONCLUSIONS}

In this paper we presented a review of the most used methods to evaluate the scattering from ocean surfaces for different applications and with different sensors. All the models require a reliable description of the ocean surface, which is mainly described in terms of its surface spectrum.

Several valuable methods have been developed, with different range of validity, simplicity, reproducibility and accuracy. Many research problems remain open, mainly related with the description of the field scattered in particular conditions, as in the case of grazing angle, which is of particular interest in coastal radar. The analysis of the existing methods evidences the fact that electromagnetic modeling is essential in order to fully comprehend the phenomena that govern the formation of the scattered field and, as a consequence, to maximize the possibility of extract physical information from the sensors that are continuously monitoring the oceans.

\section{REFERENCES}

[1] G.Franceschetti, A.Iodice, D.Riccio, G.Ruello, R.Siviero, "SAR Raw Signal Simulation of Oil Slicks in Ocean Environments", IEEE Transactions on Geoscience and Remote Sensing, vol.40, no.9, pp.19351949, 2002

[2] T. Elfouhaily, B. Chapron, and K. Katsaros, " A unified directional spectrum for long and short wind-driven waves", Journal of Geophysical Research, vol. 102, no. c7, pp. 15781-15796, 1997.

[3] G. Franceschetti and D. Riccio, Scattering, Natural Surfaces and Fractals, Academic Press, Burlington, (MA) USA, 2007.

[4] G. Franceschetti, A. Iodice, D. Riccio, "Fractal models for scattering from natural surfaces" in Scattering, edited by R.Pike and P.Sabatier, London: Academic Press, pp. 467-485, 2001.

[5] G. Franceschetti, A. Iodice, M. Migliaccio, D. Riccio, "Scattering from natural rough surfaces modelled by fractional Brownian motion twodimensional processes", IEEE Trans. Antennas Propagat., vol.47, no.9, pp. 1405-1415, 1999.

[6] A. Iodice, A. Natale, D. Riccio, "Kirchhoff Scattering From Fractal and Classical Rough Surfaces: Physical Interpretation", IEEE Transactions on Antennas and Propagation, vol.61, no.4, pp. 2156-2163, 2013.

[7] G.Franceschetti, A.Iodice, M.Migliaccio, D.Riccio, "Fractals and the Small Perturbation Scattering Model", Radio Science, vol.34, no.5, pp. 1043-1054, 1999.

[8] G.Franceschetti, A.Iodice, D.Riccio, "Scattering from Dielectric Random Fractal Surfaces via Method of Moments", IEEE Transactions on Geoscience and Remote Sensing, vol.38, no.4, pp.1644-1655, 2000.

[9] A. Iodice, A. Natale, D. Riccio, "Retrieval of Soil Surface Parameters via a Polarimetric Two-Scale Model", IEEE Trans. Geosci. Remote Sens., Vol. 49, no. 7, pp. 2531-2547, July 2011.

[10] A. Danisi, A. Iodice, D. Riccio, G. Ruello, M. Tello, J.J. Mallorqui, C. Lopez-Martinez, "SAR Simulation of Ocean Scenes Covered by Oil Slicks with Arbitrary Shapes", Proc. IGARSS '2007, Barcelona, Spain.

\section{ACKNOWLEDGMENT (Heading 5)}

The preferred spelling of the word "acknowledgment" in America is without an "e" after the "g". Avoid the stilted expression "one of us (R. B. G.) thanks ...". Instead, try "R. B. G. thanks...". Put sponsor acknowledgments in the unnumbered footnote on the first page. 